\title{
Neighbourhood Watch for the cytoskeleton
}

Recent research has shown that the two main components of the cytoskeleton, actin and microtubules, monitor each others' presence and are coordinately regulated. For example, microtubules, although they themselves have no function in generating forces during cell motility, are required for the normal actin reorganization at the leading edge of the cell. Recent evidence indicates that microtubules can even intervene with their neighbours' actions through RhoGTPases, but how was unclear. This mechanism is examined by Bokoch and colleagues on page 294 of this issue, who now show that a key regulator of Rho activity, GEF-HI, is kept in check by the microtubule network.

GEF-H1 is a nucleotide-exchange factor previously thought to activate both Rho and Rac, two small GTPases central to the regulation of actin dynamics. A clue that GEF-H1 might participate at the interface between microtubules and actin came from previous studies showing that GEF-H1 localizes to microtubules. Bokoch and colleagues set out to test the significance of this localization. To tease out what GEF-H1 does here, they asked what happens when it is overexpressed. This resulted in the stabilization of microtubules and the formation of coiled microtubule bundles similar to those induced by overexpression of microtubule-associated proteins.

At the same time, Bokoch and colleagues noticed that something else was going on - overexpression of truncated GEF-H1 also caused marked changes in cellular morphology, including the formation of actin stress fibres (see figure: actin is shown in red and overexpressed truncated GEF-H1 is shown in green. Scale bar, $20 \mu \mathrm{m})$. Interestingly, they found that the nucleotide-exchange activity was required for this effect, as was a downstream effector

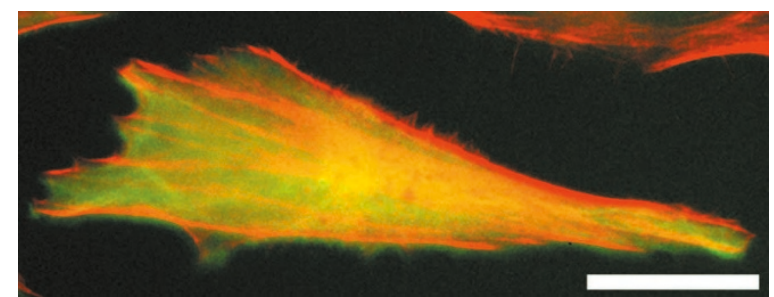

specific for Rho signalling. When they tested the ability of the truncated GEF-H1 constructs to activate downstream signalling in vivo, they saw a striking correlation between the ability of a construct to localize to microtubules and its reduced ability to activate Rho. From this they speculated that association of GEF-H1 with microtubules somehow alters GEF-H1 activity. In support of this idea, they found that depolymerizing microtubules with, for example, nocodazole, is sufficient to trigger endogenous Rho signalling - an effect that requires wild-type GEF-H1.

How microtubule binding reduces GEF-H1 activity is an open question, but it will be interesting to see whether this is a common mechanism for ensuring coordination between neighbouring cytoskeletal networks. Another process where the authors predict this will be important is during cytokinesis, where the actin-driven cleavage furrow must form near the centre of the microtubule-based spindle.

ALISON SCHULDT extracellular matrix proteins present in epithelial cell basement membranes ${ }^{17}$. Mouse embryos that are genetically deficient in $\alpha_{3} \beta_{1}$ integrin have substantially reduced viability and cells derived from these embryos fail to colonize kidney and lung. These observations suggest that this integrin is crucial for cell migration during organ development ${ }^{18}$. Interestingly, $\alpha_{3} \beta_{1}$ deficient mice also present with mild blistering of the skin, caused by the perturbation of basement membrane organization ${ }^{19}$. Given these observations, it would be useful to know if endothelial cell migration and/or proliferation is enhanced by the interaction of the HHV-8 gB envelope glycoprotein with $\alpha_{3} \beta_{1}$.

In conclusion, the growing number of bacteria and viruses that use integrins for cell entry suggests that these receptors are not simply convenient portals for cell entry, but instead have more significant functions. Integrin ligation increases the kinetics of pathogen virus internalization into cells ${ }^{8}$, a situation that would prevent virus particles from being rapidly inactivated by antibodies and/or complement. Integrin signalling could also provide a more stable environment for the microbe once it has established the infection by down-regulating the host immune response. Microbes that use $\beta_{1}$ integrins on cells of the immune system also provide an opportunity for establishing a latent infection in these cells, as well as facilitating pathogen dissemination throughout the host. Microbial pathogen spread could also be enhanced through integrin-mediated cell migration. Further studies with HHV-8, as well as other viruses, should help to increase our understanding of the precise function of integrin signalling in cell entry and microbial pathogenesis.

Glen R. Nemerow and David R. Cheresh are in the Department of Immunology, The Scripps Research Institute, La Jolla, California, 92037, USA

e-mail:gnemerow@scripps.edu
1. Cossart, P., Boquet, P., Normark, S. \& Rappuoli, R. Science 271, 315-316 (1996).

2. Akula, S. M., Pramod, N. P., Wang, F.-Z. \& Chandran, B. Cell 108, 407-419 (2002).

3. Antman, K. \& Chang, Y. New Engl. J. Med. 342, 1027-1038 (2000).

4. Akula, S. M., Wang, F. Z., Vieira, J. \& Chandran, B. Virology 282, 245-255 (2001)

5. Spear, P. G., Eisenberg, R. J. \& Cohen, G. H. Virology 275, 1-8 (2000).

6ergelson, J. M. et al. Science 275, 1320-1323 (1997).

7. Tomko, R. P., Xu, R. \& Philipson, L. Proc. Natl Acad. Sci. USA 94, 3352-3356 (1997).

8. Wickham, T. J., Mathias, P., Cheresh, D. A. \& Nemerow, G. R. Cell 73, 309-319 (1993).

9. Li, E. et al. J. Virol. 75, 5405-5409 (2001).

10. Ciarlet, M. et al. J. Virol. 76, 1109-1123 (2002).

11. Gavrilovskaya, I. N. et al. Proc. Natl Acad. Sci. USA 95, 7074-7079 (1998).

12. Li, E. et al. J. Virol. 72, 2055-2061 (1998).

13. Ireton, K. et al. Science 274, 780-782 (1996).

14. Eble, J. A. et al. Biochem. 37, 10945-10955 (1998).

15. Ganem, D. Curr. Clin. Top. Infec. Dis. 237-251 (2002).

16. Chandrasekaran, L. et al. Mol. Biol. Cell 11, 2885-2900 (2000).

17. Carter, W. G., Ryan, M. C. \& Gahr, P. J. Cell 65, 599-610 (1991).

18. Kreidberg, J. A. et al. Development 122, 3537-3547 (1996).

19. Bouvard, D. et al. Circ. Res. 89, 211-223 (2001). 